\title{
Cherry-red spot myoclonus syndrome and a-neuraminidase deficiency: neurophysiological, pharmacological and biochemical study in an adult
}

\author{
S FRANCESCHETTI, G UZIEL, S DI DONATO, L CAIMI*, AND G AVANZINI \\ From the Departments of Neurophysiology and Neurometabolic Diseases Istituto Neurologico C Besta, \\ Milan, Italy
}

SUMMARY A 22 year old patient with non-familial progressive myoclonus, macular cherry-red spot, moderate cerebellar syndrome and normal intelligence is described. The mycolonus began at the age of 18 years. Focal myoclonus could easily be elicited by voluntary and passive movements, and by touch and electrical stimulation of median nerve. Somatosensory evoked potentials showed a high voltage early component. Jerk-locked averaging of the EEG preceding action myoclonus detected an otherwise hidden, time-related, EEG spike. The myoclonus responded partially but clearly to L-5 hydroxytryptophan plus carbidopa treatment. Biochemical study showed an $\alpha$-neuraminidase deficiency in cultured fibroblasts: the decrease in this enzyme activity was compared to that found in a patient affected by mucolipidosis III.

A slowly progressing neurological syndrome that combines action sensitive and stimulus-sensitive myoclonus with cherry-red spots at the macula in patients with $\alpha$-neuraminidase deficiency has been described recently. ${ }^{1-3}$ In these patients, there were no dementia or bone deformities, and generalised seizures that occurred in two patients were easily controlled by antiepileptic therapy. According to Rapin et $a l^{2}$ this peculiar syndrome should be kept distinct from other more malignant storage disorders and progressive myoclonic epilepsies and put in a new class of diseases characterised by accumulation of sialidated glycopeptides and glycolipids in tissues, owing to a deficiency of neuraminidase. Lowden and O'Brien $^{4}$ have defined as Sialidosis type I the Cherry-red Spot Myoclonus Syndrome (CRS-MS) with normal intelligence and $\alpha$-neuraminidase deficiency.

The present report deals with electrophysiological, pharmacological and biochemical findings in a case of CRS-MS observed recently.

Address for reprint requests: Dr Silvana Franceschetti, Department of Neurophysiology, Istituto Neurologico C Besta, Via Celoria 11, 20133 Milan, Italy.

Accepted 11 April 1980

*Institute of Biochemistry, University of Milan, Italy.

\section{Case report}

The patient was an Italian male aged 21 years. There was nothing significant in the family history. The patient was cyanotic at birth but his psychomotor development had been normal. At age 13 years he had an episode of acute polyarthritis diagnosed as rheumatic fever. At age 18 years the patient began to complain of walking clumsily and a few months later he had a short tonic-clonic generalised seizure. Neurological examination performed at that time showed mild dysmetria and brisk tendon reflexes.

In the following months his difficulties in standing and walking rapidly became worse with the appearance of muscular jerks that affected all four limbs and were exacerbated by voluntary movement. A progressive irregular course eventually led to a condition in which the patient was no longer able to care for himself. When he was admitted to hospital in December 1978 (at the age of 21 years) neurological examination showed diffuse hypotonia, brisk deep reflexes and bilateral Babinski signs. A mild ataxia was evident on finger-nose and heel-knee tests, although evaluation was made difficult by the superimposed myoclonic jerks. The myoclonic jerks were absent at rest but precipitated by any attempt to change posture or even by a simple request to move. The focal character of the myoclonus was evident only at the beginning of the voluntary movement owing to its marked tendency to spread rapidly and end up in repeated massive bilateral 
jerks. In addition to the action and intention myoclonus, there was also reflex myoclonus that could be triggered by passive movements and tactile stimulation. Examination of the ocular fundus showed a normal optic disk and a cherry-red spot in the macular region, more striking in the right eye (fig 1). The results of audiometric examination were normal. The patient was still able to communicate satisfactorily with other people, talking very slowly and using limited mimic movements. He showed no sign of mental impairment.

The CT scan was normal. Blood examination (red and white cell count, erythrocyte sedimentation rate, electrolytes, nitrogen, glucose and bilirubin levels, SGOT, SGPT, alkaline phosphatase, serum protein electrophoresis, serum immunodiffusion) was normal. In the cerebrospinal fluid cell count, total protein, glucose, chloride, and protein electrophoresis and immunodiffusion were normal; 5-hydroxyindoleacetic acid level was $35.57 \mathrm{ng} / \mathrm{ml}$ (controls mean value $31 \cdot 37+4 \cdot 43$ ). Blood and urinary aminoacids were normal. Assays of arylsulphatase $\mathrm{A}$ and $\beta$-galactosidase in the leucocyte also were normal. Electrophysiological findings The EEG and EMG were recorded from surface electrodes on a 12 channel OTE Biomedica polygraph. Scalp electrodes were placed according to the 10-20 international system. In addition selected recording samples were stored on FM magnetic tape for averaging of EEG and EMG activity, time-locked to stimuli and to myoclonic jerks (PDP 11/34 computer system), and for spectral analysis of EEG (OTE Biomedica BergFOURIER Analyzer). For detection of non-obvious EEG correlates of myoclonus, the EEG activity preceding the myoclonic discharge was analysed by jerk-locked averaging employing a $100 \mathrm{~ms}$ analogue delay-line circuit. An electronic window (Vescovini

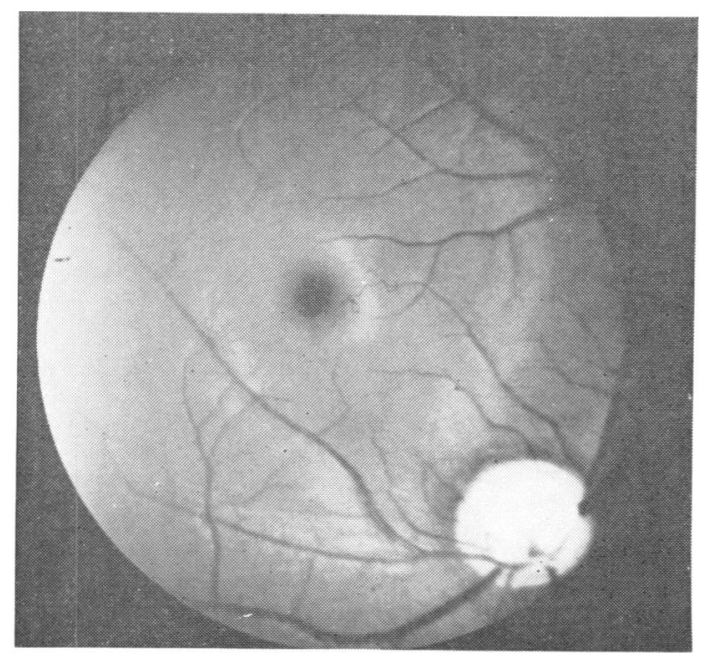

Fig 1 Fundus oculi (right eye): note macular cherry-red spot (courtesy of Dr Delle Grottaglie).
477-25-28) was employed to trigger the program at the onset of myoclonic potentials. One hundred and twenty EEG samples of $250 \mathrm{~ms}$ each were usually averaged.

Somatosensory evoked potentials (SEPs) and reflex myoclonus were elicited by median nerve stimulation by paired surface electrodes placed $3 \mathrm{~cm}$ apart on the wrist. Square wave pulses of $1 \mathrm{~ms}$ duration were delivered at rate of $0 \cdot 5-1 \mathrm{~s}$, the intensity was adjusted to induce the least detectable motor response of the opponens muscle. The SEP values computed for 200 bipolar EEG samples were recorded from central and parietal (C3-P3) regions; the analysis time was $250 \mathrm{~ms}$. Evoked EMG activity (that is reflex myoclonus) was displayed on the oscilloscope and fed into the computer. Sixty responses were usually averaged (analysis time $250 \mathrm{~ms}$ ).

In the resting condition, the electrophysiological recording did not show anything of particular interest. Voluntary and passive movements and tactile stimulation by light touch or tap precipitated vigorous focal myoclonic jerks consisting of repetitive biphasic or polyphasic EMG potential lasting 15-30 $\mathrm{ms}$, occurring five to 15 times per second, which spread rapidly to all the muscles examined eventually leading to a recurrent massive myoclonus (fig 2 ). Both agonist and antagonist muscles usually were involved. Concomitant EEG bursts of $16-20 \mathrm{~Hz}$ activity were almost always detectable in central and parietal regions of both sides, in spite of the superimposed muscle artifacts.

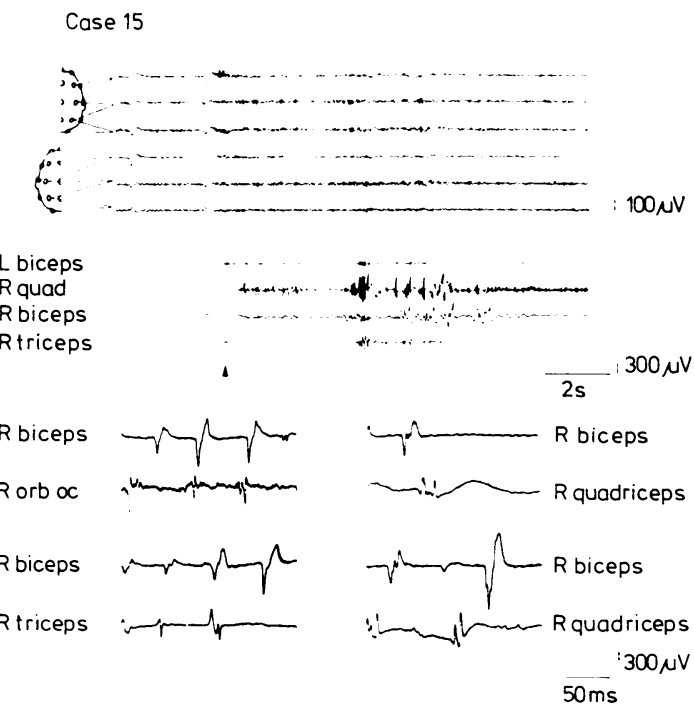

Fig 2 Top: EEG-EMG recording of action myoclonus evoked by voluntary movement of lower limbs. Bottom: EMG recording of action myoclonus from paired muscles. Note that the biceps could lead or follow the quadriceps according to whether it was precipitated by voluntary movement of upper limbs (upper traces) or of lower limbs (lower traces). 
The characteristics of the EEG correlate were demonstrated better by jerk-locked averaging which revealed that each jerk was time-related to an EEG spike lasting about $30 \mathrm{~ms}$ and preceding the muscle potential in the contralateral biceps by $17 \mathrm{~ms}$ (fig 3A).

Reflex myoclonus was regularly elicited by electrical stimulation of the median nerve as shown in fig 3B. The EMG recording in this case showed a more complex wave form than that of action myoclonus and with longer duration $(40-60 \mathrm{~ms})$. A quite constant pattern of latency values ranging from 35 ms (for proximal muscles of the ipsilateral upper limb) to $53 \mathrm{~ms}$ (for ipsilateral quadriceps) was observed, as demonstrated by the averaging of 60 responses (fig 4). Note in the same figure that the contralateral SEP evoked by the same median nerve stimulation shows an high voltage $(35 \mu \mathrm{V})$ early component with $21.5 \mathrm{~ms}$ latency preceding by $15 \mathrm{~ms}$ the EMG potential recorded in the biceps muscle.

Pharmacological tests and therapy Pharmacological tests with L-5-hydroxytryptophan (L-5HTP) and clonazepam (CZP) were performed according to a protocol previously set up in our department. ${ }^{5}$ L-5HTP (Roche: Ro 3-5940/601) was administered by intravenous (iv) infusion of $100 \mathrm{mg}$ in $250 \mathrm{ml}$ of isotonic saline solution (infusion time $60 \mathrm{~s}$ ) after pretreatment with carbidopa (Merck Sharpe and Dohme) $100 \mathrm{mg}$ given by mouth.

Thirty minutes after the start of the infusion a clearcut reduction of the action myoclonus (fig 5) and the reflex myoclonus was observed resulting in marked functional improvement. This beneficial effect lasted four hours. No side effects were observed. A similar but more transient effect was induced by a single $1 \mathrm{mg}$ dose of CZP given intravenously.

A chronic trial was then started with increasing oral doses of L-5HTP plus carbidopa, up to 600 and $125 \mathrm{mg}$ respectively. These two drugs were simply added to the preceding therapy, which was maintained unchanged. During 12 months of follow-up, the beneficial effects of L-5HTP on action-sensitive and stimulus-sensitive myoclonus were confirmed. The ensuing improvement in motor performance increased the degree of self-sufficiency of the patient in some daily activities such as dressing, washing and eating. So far there has been no sign of loss of efficacy. Neither clinical nor laboratory side effects have been observed.

Biochemical findings Fibroblasts from normal controls, a patient with mucolipidosis III (ML III) and the CRS-MS patient were grown in Eagle's MEM, supplemented with $10 \%$ foetal calf serum as previously described. ${ }^{6}$ Cells were analysed for lysosomal enzymes and $\alpha$-neuraminindase activities after five to 15 subcultures, seven days after they became confluent.

Lysosomal enzymes in cultured cells and in culture medium were analysed as follows: (a) cells were washed twice with tris-buffered saline (TBS), $\mathrm{pH} 7 \cdot 4$ and trypsinised with $0.25 \%$ trypsin- $1.0 \mathrm{mM}$ EDTA,
pH 7•4. The cell pellets were washed twice with TBS. Lysosomal enzymes activities in sonicated cells were then analysed as previously described ${ }^{7}$; (b) fibroblasts were washed twice with Eagle's MEM without foetal calf serum and then incubated overnight with MEM in order to remove serum proteins entrapped in the cell network. The medium was removed and the fibroblasts incubated for $24 \mathrm{hrs}$ at $37^{\circ}$ in MEM: the

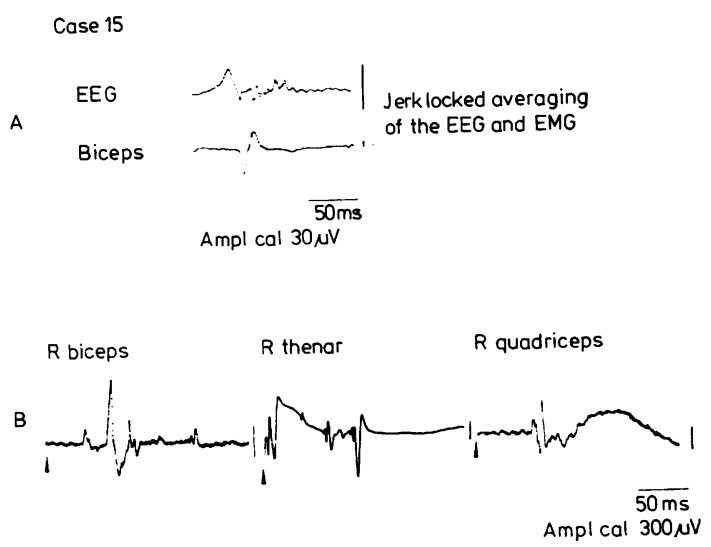

Fig 3 A: Jerk-locked averaging of the EEG and EMG during action myoclonus showing an EEG spike preceding the muscolar potential by $17 \mathrm{msec}$. $B$ : Reflex myoclonus evoked by stimulation of right median nerve at the wrist.

Case 15

$$
\text { Median N stim - averaging }
$$

L sep

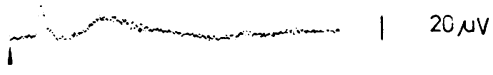

R trapezius

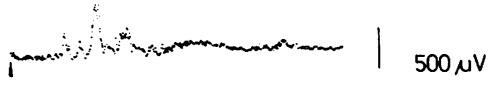

$\mathrm{R}$ biceps

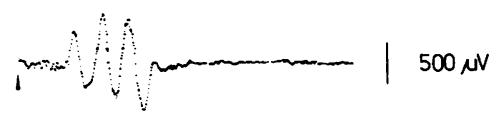

$R$ thenar

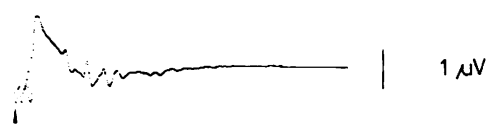

R quadriceps

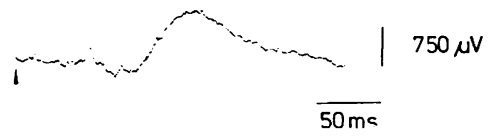

Fig 4 Averaging of reflex myoclonus evoked in different muscles by stimulation of the right median nerve at the wrist is compared with contralateral $E E G$ response evoked by the same stimulation (EMG averaging: 60 samples; $E E G$ averaging: 200 samples, bipolar recording from C3-P3 leads). 
CASE 15

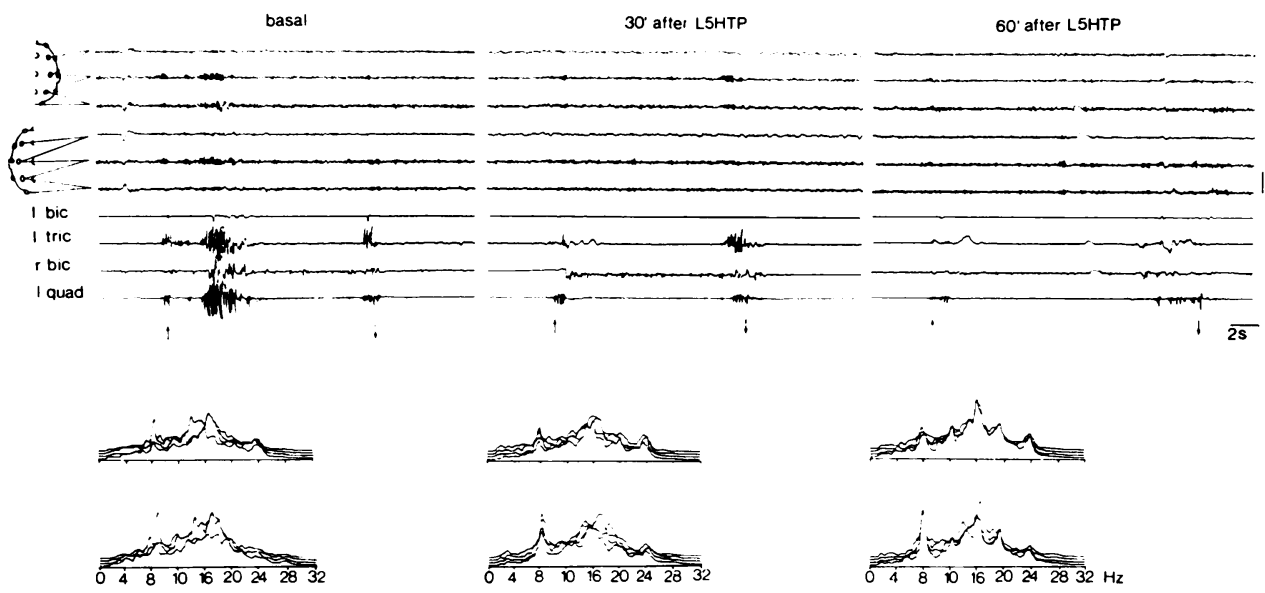

Fig 5 Effect of L5HTP infusion on action myoclonus evoked by voluntary activation of muscles of lower limbs. At bottom of figure: power spectra computed on F3-C3 (upper trace) and C3-P3 (lower trace), showing no remarkable changes in EEG frequency composition. Each trace displays the composition of 30 sec sample.

media from three plates for each cell line were pooled: lysosomal enzymes secreted by cultured fibroblasts were precipitated with $80 \% \quad\left(\mathrm{NH}_{4}\right)_{2} \mathrm{SO}_{4}$ centrifuged at $3000 \mathrm{rpm}$ at $4^{\circ} \mathrm{C}$ in the presence of $0.43 \mathrm{mg} / \mathrm{ml}$ albumin as a carrier. The precipitates were suspended in $1 \mathrm{ml}$ of glass redistilled water, dialysed for $24 \mathrm{hr}$ against $500 \mathrm{ml}$ of $0.5 \mathrm{M}$ acetate buffer pH $5 \cdot 5$, in $0 \cdot 15 \mathrm{M} \mathrm{NaCl}$, with two changes of dialysis solution, and then analysed for lysosomal enzymes.

Neuraminidase activity in sonicated fibroblasts was determined under optimal conditions with $\alpha-\mathrm{N}-$ acetyl-neuraminylactose $(\alpha 2-3)$ as substrate as recently described. ${ }^{8}$ Table 1 shows the activities of $\alpha$-neuraminidase in the cells of controls, of the ML III patient and of the CRS-MS patient: $\alpha$-neuraminidase was markedly reduced, to about $5 \%$ of the mean control values in the cells of the two patients.

Table 2 shows the activities of five lysosomal hydrolases in cultured fibroblasts and in the culture media from the controls and the two patients. A marked reduction of the activity of the five hydrolases was observed in the cells of the ML III patient, with corresponding increases in enzyme activity in his culture medium, except for that of $\beta$-galactosidase; this latter enzyme and the other four hydrolases measured in the cells and in the culture medium of CRS-MS patient showed activities comparable to those of controls.

Table 1 Activity of $\alpha$-neuraminidase in cultured fibroblasts from the two patients and the controls

\begin{tabular}{lc}
\hline & $\alpha-$ neuraminidase activity \\
\hline Controls (15) & $0.300 \pm 0.006$ \\
Patient CRS-MS & 0.020 \\
Patient ML III & 0.012 \\
(Mucolipidosis III) &
\end{tabular}

Activity is expressed as nanomoles $\alpha-\mathrm{N}$-acetyl-neuraminilactose ( $\alpha$ 2-3) hydrolysed $/ \mathrm{mg} / \mathrm{protein} / \mathrm{hr} \pm \mathrm{SE}$. In brackets the number of control cell lines in duplicate.

Table 2 Activity of lysosomal hydrolases in cultured cells and in culture medium of cells from the patients and the controls*

\begin{tabular}{|c|c|c|c|c|c|c|}
\hline & \multicolumn{2}{|l|}{ Controls } & \multicolumn{2}{|c|}{$C R S-M S$ patient } & \multicolumn{2}{|c|}{$M L I I I$ patient } \\
\hline & Cells & Medium & Cells & Medium & Cells & Medium \\
\hline $\begin{array}{l}\text { arylsulphates A } \\
\beta \text {-hexosaminidase } \\
\alpha \text {-mannosidase } \\
\alpha \text {-fucosidase } \\
\beta \text {-galactosidase }\end{array}$ & $\begin{array}{l}1240 \pm 436(12) \\
8921 \pm 2610(12) \\
259 \pm 64(6) \\
217 \pm 72(6) \\
724 \pm 180(12)\end{array}$ & $\begin{array}{c}16.5 \pm 2.6(3) \\
87 \pm 21(3) \\
0.93 \pm 0.16(3) \\
2 \cdot 5 \pm 0.4(3) \\
1 \cdot 29 \pm 0.26(3)\end{array}$ & $\begin{array}{r}1832 \\
11704 \\
239 \\
229 \\
1227\end{array}$ & $\begin{array}{l}20 \cdot 6 \\
102 \\
0 \cdot 20 \\
1 \cdot 7 \\
0 \cdot 75\end{array}$ & $\begin{array}{c}161 \\
2505 \\
0 \\
19 \cdot 1 \\
144\end{array}$ & $\begin{array}{c}59 \cdot 2 \\
324 \\
19 \cdot 6 \\
4 \cdot 8 \\
1 \cdot 37\end{array}$ \\
\hline
\end{tabular}

*Activities are expressed as nanomoles substrates hydrolised/mg protein/hr \pm S.D. In brackets the number of lines examined in duplicate. 


\section{Comment}

The clinical picture of this patient is strikingly similar to that reported by Rapin et al. ${ }^{2}$ Common to our patient and to the three previously reported cases $^{2}$ are: (1) progressive action and stimulus-sensitive myoclonus with late onset, (2) cherry-red spot at the macula, (3) deficiency of $\alpha$-neuraminidase, (4) no evidence of dementia. Our patient shares with the two sisters reported by Rapin et $a l^{2}$ an Italian origin.

According to Engel et al, ${ }^{9}$ the electrophysiological findings in this syndrome include: (1) low voltage fast EEG background activity; (2) paroxysmal bursts of bilateral $10-20 \mathrm{~Hz}$ positive spikes, time-locked but not topographically related to the myoclonus; (3) changes in SEP amplitude and waveform. Similar but not identical features were present in our patient who also showed a low voltage fast EEG background with paroxysmal bursts which can be better defined as "central fast rhythm" according to Kelly et $a l^{10}$ rather than as $10-20$ positive spikes.

By the jerk-locked averaging technique, however, it was possible to detect a time-locked EEG spike preceding each myoclonic jerk. According to Shibasaki et al, ${ }^{11}$ this myoclonus-related cortical spike may be due to the same mechanisms underlying the giant primary SEP (also present in our patient). Whether or not these two phenomena can be related to a cortical reflex type of myoclonus is not specifically relevant to the present discussion: the question of corticalversus-subcortical generators of myoclonus will be discussed in more detail in another paper now in preparation, based on a large number of patients with various kinds of myoclonus.

An interesting point is the effect of L 5HTP on action-sensitive and stimulus-sensitive myoclonus in our patient, both in an acute and a chronic trial. Beneficial effects of serotonin precursors have been demonstrated far less certainly in non-postanoxic myoclonus syndromes than in postanoxic ones. ${ }^{12-14}$ It has been suggested that low CSF concentrations of 5HIAA (which suggests an impairment in serotonin metabolism) may be used to predict the efficacy of serotonin precursors and that this criterion could be used to detect an L 5HTP sensitive population among patients with myoclonic syndromes. ${ }^{13}$ This would not hold true for our patient or for some other patients with myoclonic syndromes we have studied. According to the preliminary results of our study the only common characteristic shared by all patients responding to L 5HTP was the prominence of action and intention-myoclonus.
It should be noted, however, that such myoclonus also was present in the three patients with a cherry-red spot reported by Rapin et $a l^{2}$ to be unresponsive to L 5HTP plus carbidopa.

$\alpha$-neuraminidase deficiency in cultured fibroblasts from patients with mucolipidoses II and III, ${ }^{15} 16$ has been reported. In these patients, $\alpha$ neuraminidase deficiency accompanied marked deficiencies in several lysosomal hydrolases in cultured cells, with increases in the corresponding activities in the cell culture media or serum. ${ }^{1718}$ In cultured fibroblasts from patients with mucolipidosis I, a disease characterised by bone dysplasia, Hurler-life face, macular cherryred spot, myoclonic jerks, progressive dementia and no inclusion bodies in cultured cells, the marked deficiency in $\alpha$-neuraminidase is not associated with other known enzyme deficiencies. ${ }^{1 i}$ Neuraminidase deficiency has been regarded as a primary defect in this condition and the disease named "Sialidosis." 2021

The spectrum of $\alpha$-neuraminidase deficiency was broadened by the reports of Rapin et $a l^{2}$ and O'Brien, ${ }^{1}$ when they described their three patients with progressive incapacitating myoclonus, macular cherry-red spots and $\alpha$-neuraminidase deficiency in their cultured cells. These patients differed from those with ML I in the adult onset of the signs and symptoms, the lack of bone dysplasia and the normal intelligence. They share with the ML I patient macular cherry-red spots, myoclonus and $\alpha$-neuraminidase deficiency. ${ }^{2}$ Moreover, additional patients with clinical features distinct from those of the patients with mucolipidoses I, II, III and the cherry-red spot myoclonus syndrome, but with $\alpha$-neuraminidase deficiency, have been reported: an eight-month-old girl with normal mental development and dysostosis multiplex ${ }^{10}$ and an adult patient with skeletal dysplasia, cerebellar ataxia, cherry-red spot and myoclonus ${ }^{22}$ : conversely, patients with a phenotypic condition similar to mucolipidosis I and to the CRS-MS have been described in whom the biochemical defect was however $\beta$-galactosidase deficiency ${ }^{23-25}$ : in some patients $\beta$-galactosidase deficiency was associated and probably secondary to $\alpha$-neuraminidase deficiency. ${ }^{26-28}$ It is clear, therefore, that similar phenotypic conditions can be associated with different biochemical abnormalities and that a given enzyme deficiency (for example, $\alpha$ neuraminidase deficiency) can be associated with different clinical phenotypes.

Our patient was clinically and biochemically closely similar to the patients described by Rapin et $a l^{2}$ and falls into the type I Sialidosis. ${ }^{4}$ 
The degree of $\alpha$-neuraminidase deficiency in cultured cells from our patient, as measured with $\alpha-2-3$ neuraminylactose, was not distinguishable from that found in cultured cells of a patient with ML III (table 2). The two patients could not be distinguished on the basis of $\alpha$-neuraminidase activity in their cultured cells. However, the two diseases are clinically and biochemically different; cultured cells from the CRS-MS patient showed both intracellular and extracellular activities of lysosomal enzymes comparable to those of controls, while cells from ML III patient showed the known ${ }^{18}$ decrease in intracellular and increase in extracellular lysosomal enzyme activities (table 2).

$\alpha$-neuraminidase deficiency is a secondary defect in I-cell disease, ${ }^{23} 29$ but it is considered a primary defect in both mucolipidosis I and the cherry-red spot myoclonus syndrome. We believe, however, that a more detailed characterisation of $\alpha$-neuraminidase activity using natural substrates $^{8}$ is needed in order to link well-defined clinical conditions with biochemical abnormalities.

We thank Marisa Andreasi and Marilena Scarpina for technical assistance. L 5HTP was supplied by Roche Company and Carbidopa by Merck Sharpe and Dohme.

\section{References}

1 O'Brien JS. Neuraminidase deficiency in the cherry-red spot myoclonus syndrome. Biochem Biophys Res Commun 1977; 79:1136-41.

2 Rapin I, Goldfisher S, Ketzman R, Engel J, O'Brien JS. The cherry-red spot myoclonus syndrome. Ann Neurol 1978; 3:234-42.

3 Thomas PK, Abrams JD, Dallas Swallow, Stewart G. Sialidosis type I: cherry-red spot myoclonus syndrome with sialidose deficiency and altered electrophoretic mobilities of some enzymes known to be glycoproteins. I. Clinical findings. J Neuro! Neurosurg Psychiatry 1979; 42:873-80.

4 Lowden JA, O'Brien JS. Sialidosis: a review of human neuraminidase deficiency. Am J Hum Genet 1979; 31:1-18.

5 Avanzini G, Bossi L, Caraceni $\mathrm{T}$ et al. Effect of L-5HTP and drugs acting on serotonin metabolism in various myoclonic syndromes. In: Majkowski J, ed. Epilepsy-Clinical and experimental research. Warsaw: Pol Chapt Internat League against Epilepsy, 1979: in press.

6 Di Donato S, Wiesmann UN, Herschkowitz $N$. Membrane absorption and internalisation of $\left(\mathrm{C}_{14}\right)$ chloroquine by cultured human fibroblasts Biochem Pharmacol 1977; 26:7-10.

7 Tettamanti G, Lombardo A, Marchesini S et ol. Lipid storage diseases: biochemical diagnosis. Biochem Exp Biol 1977; 13:45-60.
8 Caimi L, Lombardo A, Preti A, Wiesmann UN, Tettamanti G. Optimal conditions for the assay of fibroblast neuraminidase with different natural substrates. Biochim Biophys Acta 1979; 571: 137-46.

9 Engel J, Rapin L, Giblin DR. Electrophysiological studies in two patients with cherry-red spot myoclonus syndrome. Epilepsia 1977; 18: 73-87.

10 Kelly JJ Jr, Sharbrough FW, Westmoreland BF. Movement-activated central fast rhythms: an EEG finding in action myoclonus. Neurology 1978; 28:1037-40.

11 Shibasaki H, Yamashita Y, Kuroiwa Y. Electroencephalographic studies of myoclonus. Brain 1978; 101:447-60.

12 Lhermitte F, Peterfalvi M, Mareau R, Gazengel J, Serdaru M. Analyse pharmacologique d'un cas de myoclonies d'intention et d'action postanoxiques. Rev Neurol (Paris) 1971; 124:21-31.

13 Chadwick D, Hallett $M$, Harris $R$, Jenner $P$, Reynolds EH, Marsden CD. Clinical, biochemical and physiological features distinguishing myoclonus responsive to 5-hydroxytryptophan with a monoamine oxidase inhibitor and clonazepam. Brain 1977; 100:455-78.

14 Van Woert MH, Rosenbaum D, Howieson I, Bowers MB. Long-term therapy of myoclonus and other neurologic disorders with L-5-Hydroxytryptophan and Carbidopa. N Engl J Met 1977; 296:70-5.

15 Stretcher G, Michalski JC, Montrenil J, Tarriaux JP. Deficit in neuraminidase associated with mucolipidosis II (I-cell disease). Biomedicine 1976; 25:238-40.

16 Thomas GH, Tiller GE, Reynolds LW, Miller CS, Bace JW. Increased levels of sialic acid associated with a sialidase deficiency in I-cell disease mucolipidosis II) fibroblasts. Biochem Biophys Res Commun 1976; 71:188-95.

17 Hickman S. Neufeld EF. A hypothesis for I-cell disease: Defective hydrolases that do not enter lysosomes. Biochem Biophys Res Commun 1972; 49:992-9.

18 Wiesmann UN, Herschkowitz NN. Studies on pathogenetic mechanism of I-cell disease in cultured fibroblasts. Pediatr Res 1974; 8:865-70.

19 Cantz M, Gehler J, Spranger J. Mucolipidosis I: Increased sialic acid content and deficiency of an $\alpha$-N-acetylneuraminidase in cultured fibroblasts. Biochem Biophys Res Commun 1977; 74: 732-8.

20 Spranger J, Gehler J, Cantz M. Mucolipidosis I. a Sialicidosis. Am J Med Genet 1977; 1:21-9.

21 Durand P, Gatti R, Cavalieri S et al. Sialidosis Mucolipidosis I). Helv Paediatr Acta 1977; 32: 391-400.

22 Kelly TE, Graetz G. Isolated acid neuraminidase deficiency: a distinct lysosomal storage disease. Am J Med Genet 1977; 1:31-46.

23 Miyatrake T, Yamada T, Serzuki M et al. Sialidase deficiency in adult type neuronal storage 
disease. FEBS Letters 1979; 97:257-9.

24 Suzuki Y, Nakamura N, Shimade Y, Yotsuinoto $H$, Endo $H$, Nagashima K. Macular cherry-red spot and $\beta$-galactosidase deficiency in an adult. Arch Neurol 1977; 34:157-61.

25 Orii $\mathrm{T}$, Minami $\mathrm{R}$, Sukegawa $\mathrm{K}$ et al. A new type of mucolipidosis with $\beta$-galactosidase deficiency and glycopeptiduria. Tohoku J Exp Med $1972 ; 107: 303-15$.

26 Yamamoto A, Adachi S, Kawamura S et al. Localised $\beta$-galactosidase deficiency. Occurrence in cerebellar ataxia with myoclonus epilepsy and macular cherry-red spot-a new variant of $\mathrm{GM}_{1}$-gangliosidosis? Arch Intern Med 1974; 134: 627-34.

27 Thomas GH, Goldberg ME, Miller CS, Reynolds LW. Neuraminidase deficiency in the original patient with the Goldberg syndrome. Clin Genet 1979; 16:323-30.

28 Okada S, Yutaka T, Tomochika $\mathrm{K}$ et al. A case neuraminidase deficiency associated with a partial $\beta$-galactosidase defect. Eur J Pediatr 1979; 130:239-49.

29 Wenger DA, Tarby TJ, Wharton C. Macular cherry-read spot and myoclonus with dementia: coexistent neuraminidase and $\beta$-galactosidase deficiencies. Biochem Biophys Res Commun 1978; 82:589-95.

30 Di Donato S. Biochemistry of mucolipidosis II, or I-cell disease. In: Berra B, Balduini B, Di Donato S, Tettamanti G, eds. Perspectives in Inherited Metabolic Disease, vol 2, Glycoproteins and Glycosaminoglycans in Health and Disease. 1979: 129-39. 